\title{
417 - Olfactory signatures in models of aging and Alzheimer's disease and the effect of social isolation: A translational neuroscience approach in times of coronavirus pandemic (COVID-19).
} $\underline{\text { Daniela Marín-Pardo }}^{1,2}$; Lydia Gimenez-Llort $^{1,2}$

${ }^{1}$ Department of Psychiatry and Forensic Medicine, School of Medicine, Universitat Autònoma de Barcelona, Barcelona, Spain

${ }^{2}$ Institute of Neuroscience, School of Medicine, Universitat Autònoma de Barcelona, Barcelona, Spain

${ }^{3}$ Envellir bé - Saber Envejecer - Healthy Aging Charity Organization, Sant Quirze del Vallès, Barcelona, Spain

Sensory systems ensure the ability to perceive and recognize the world. Therefore, the temporal course and the severity of their involution through the aging process can be critical. In the elderly, sensory impairments significantly increase their risk of biological, psychological and social impoverishment. Olfactory loss, known to happen in bacterial and viral infections and considered an early biomarker in Alzheimer's and Parkinson's diseases neurodegenerative processes, has been reported also as an early indicator of current infection by SARS-CoV-2. At the translational level, in the present work, we have studied olfactory ethograms in normal and advanced AD-related pathological aging using wildtype and the 3xTg-AD mice, a genetic model of Alzheimer's disease that presents AD-cognitive dysfunction but also a conspicuous BPSD-like phenotype. An olfactory paradigm, involving the equivalent to one day food deprivation, was used to investigate in middle-aged males and females with normal and ADpathological aging the ethological patterns shown in the olfactory inspection of a new cage with beddings and the posterior detection, finding and consumption of food pellets hidden in this new anxiogenic environment. Males with normal and pathological aging were equally delayed in their first contact with food pellets, while in female sex this latency was dependent on the genotype (longer in 3xTg-AD mice, shorter in those with normal aging). Once the animals had inspected the arena, the latencies to smell, find and eat the food pellets were found progressively increased in males with normal aging, but consecutively developed in 3xTg-AD mice. In contrast, both groups of females exhibited longer delays as compared to males, and the temporal pattern of their ethogram to smell-find-eat the food was faster. In 3xTg-AD males, social isolation (naturally occurring due to death of counterparts) emphasized these olfactory patterns, which were independent of the punctual loss of weight of this paradigm. The results show that this paradigm provides distinct contextual, sex and genotype olfactory ethogram signatures useful to investigate olfactory function in normal and AD-pathological aging. Also, that isolation has an impact enhancing the changes in the olfactory signature here described, for the first time, in the 3xTg-AD mice model of Alzheimer's disease. 